Provided for non-commercial research and educational use only. Not for reproduction or distribution or commercial use.

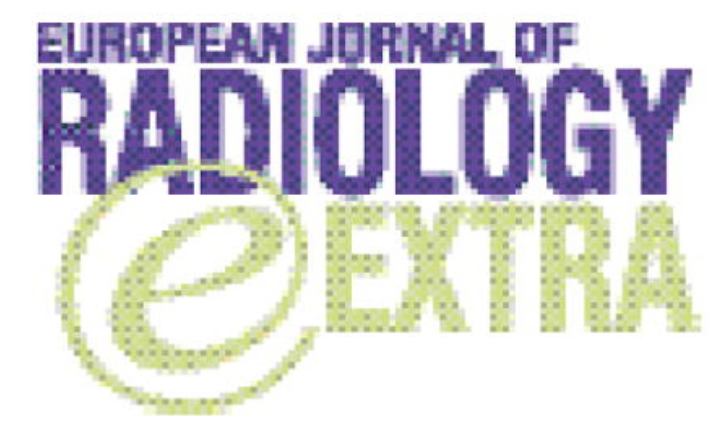

This article was originally published in a journal published by Elsevier, and the attached copy is provided by Elsevier for the author's benefit and for the benefit of the author's institution, for non-commercial research and educational use including without limitation use in instruction at your institution, sending it to specific colleagues that you know, and providing a copy to your institution's administrator.

All other uses, reproduction and distribution, including without limitation commercial reprints, selling or licensing copies or access,

or posting on open internet sites, your personal or institution's website or repository, are prohibited. For exceptions, permission may be sought for such use through Elsevier's permissions site at: 


\title{
Brain injury due to persistent hyperinsulinemic hypoglycemia of infancy
}

\author{
Pieter Julien Luc De Visschere ${ }^{\mathrm{a}, *}$, Patrick Seynaeve ${ }^{\mathrm{a}}$, \\ Frederik Vanrietvelde ${ }^{\mathrm{a}}$, Maarten Senepart ${ }^{\mathrm{b}}$ \\ a Department of Radiology and MR Imaging, AZ Groeninge Hospital, Loofstraat 43, 8500 Kortrijk, Belgium \\ b Department of Paediatrics, AZ Groeninge Hospital, Kortrijk, Belgium
}

Received 19 July 2006; received in revised form 13 January 2007; accepted 15 January 2007

\begin{abstract}
We report a case of brain damage in a 6-year old boy with seizures. On admission the patient presented with seizures two to three times a day since 1 year. Shortly after birth the boy was diagnosed with persistent hyperinsulinemic hypoglycemia of infancy. We describe the magnetic resonance imaging findings of brain lesions in our patient and correlate these to the literature. PHHI is a very uncommon disease although it is probably the most frequent cause of persistent neonatal hypoglycaemia. The disease is often associated with brain lesions, showing a rather typical distribution pattern, affecting most severely the parietal and occipital lobes of the cerebral hemispheres.
\end{abstract}

(c) 2007 Elsevier Ireland Ltd. All rights reserved.

Keywords: Infancy; MRI; Hypoglycemia; Brain

\section{Introduction}

Persistent hyperinsulinemic hypoglycemia of infancy (PHHI) is a rare disorder in which insulin secretion in the neonatal period is excessive and glucose-independent, resulting in severe bouts of hypoglycaemia. It is a very uncommon disease although it is probably the most frequent cause of neonatal hypoglycaemia [1-4]. The underlying pathomechanism is inappropriate oversecretion of insulin, leading to severe hypoglycaemia. The disease was previously known as nesidioblastosis, a term first used by Laidlaw in 1938 to describe neodifferentiation of pancreatic islets, changes seen in hyperfunctional disorders of pancreatic insulin-producing cells [5]. Nowadays this term has been replaced by persistent hyperinsulinemic hypoglycemia of infancy or congenital hyperinsulinism of the newborn. It is a potentially lifethreatening condition. The disease is often associated with brain atrophy and important permanent brain damage, which have been attributed to periods of hypoglycaemia. On MRI

\footnotetext{
* Corresponding author. Tel.: +32 478790144 .

E-mail address: pmzgvm@yahoo.com (P.J.L. De Visschere).
}

hypoglycaemic damage has a typical distribution pattern, affecting most severely the parietal and occipital lobes of the cerebral hemispheres $[3,4,6,7]$. The importance of early diagnosis and appropriate management cannot be overemphasized because neuroglycopenia in association with PHHI is a preventable cause of neurological handicap. We present a case of a child in whom brain lesions are documented at the age of 6 years after developing episodes of convulsions at the age of 5 .

\section{Case report}

A 6-year-old boy was referred to the department of radiology for cranial magnetic resonance imaging investigation. He presents with episodes of convulsions two to three times a day, developed since 1 year. The seizures manifest as focal jerking of the left leg and sometimes the left arm without losing consciousness.

He was born at 37.5 weeks after an uneventful pregnancy and delivery. There was an initial normal postnatal course but shortly after birth the boy had repeated, profound hypoglycaemic episodes. Insulin levels were obtained 
and showed marked elevation, establishing the diagnosis of persistent hyperinsulinemic hypoglycemia of infancy. Pancreatic venous sampling was consistent with diffuse form of PHHI. Treatment included intravenous glucagon and octreotide. Diazoxide did not result effect. Because the hypoglycaemic episodes had not abated, the infant underwent a subtotal pancreatectomy. Insulin levels at present are satisfying. Octreotide (sandostatin) 3/day SC remains necessary to stabilize serum glucose levels with normal glucose uptake. Because of insufficient feeding pattern a gastrostomy was inevitable. The convulsions are treated with tegretol sirup in combination with depakine. No initial MR neuroimaging studies were obtained in the acute phase.

Actual physical examination reveals normal growth and weight for his age. Neurological examination shows a poor equilibrium and a weak coordination and locomotor system. Initially there was a normal mental and motor development, but meanwhile he attends special education because of locomotor difficulties.

Electric encephalographic investigation shows regular epileptiformic grapho-elements, in both centroparietal lobes and on the right side in the temporo-occipital lobes.

On axial FSE T2-weighted MR-images (Figs. 1-3) areas of abnormal high signal intensity are present in the deep and subcortical periventricular white matter in the occipital and parietal lobes. The affected areas demonstrate hypointensities on axial SE T1-weighted MR-images. (Fig. 4) At the same level there is marked thinning of the occipital and parietal

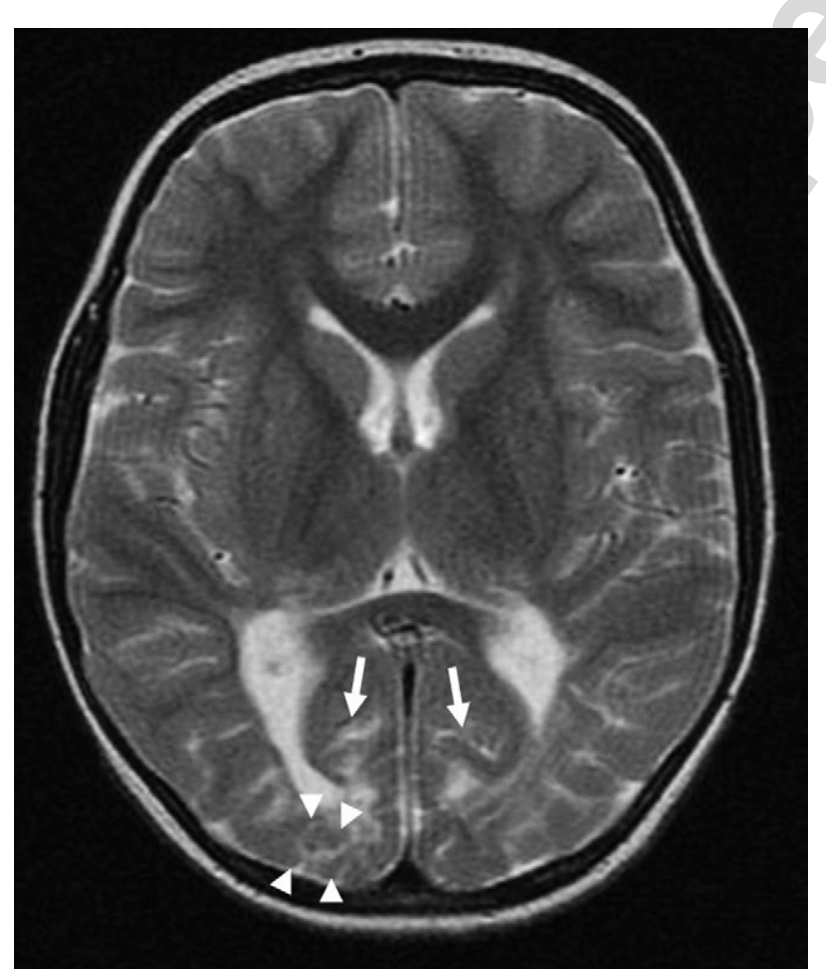

Fig. 1. Axial FSE T2 MRI. Areas of abnormal high signal intensity on T2 weighted MR images are seen in the deep and subcortical periventricular white matter of the occipital and parietal lobes (arrows). There is marked thinning of the occipital and parietal cortex (arrowheads).

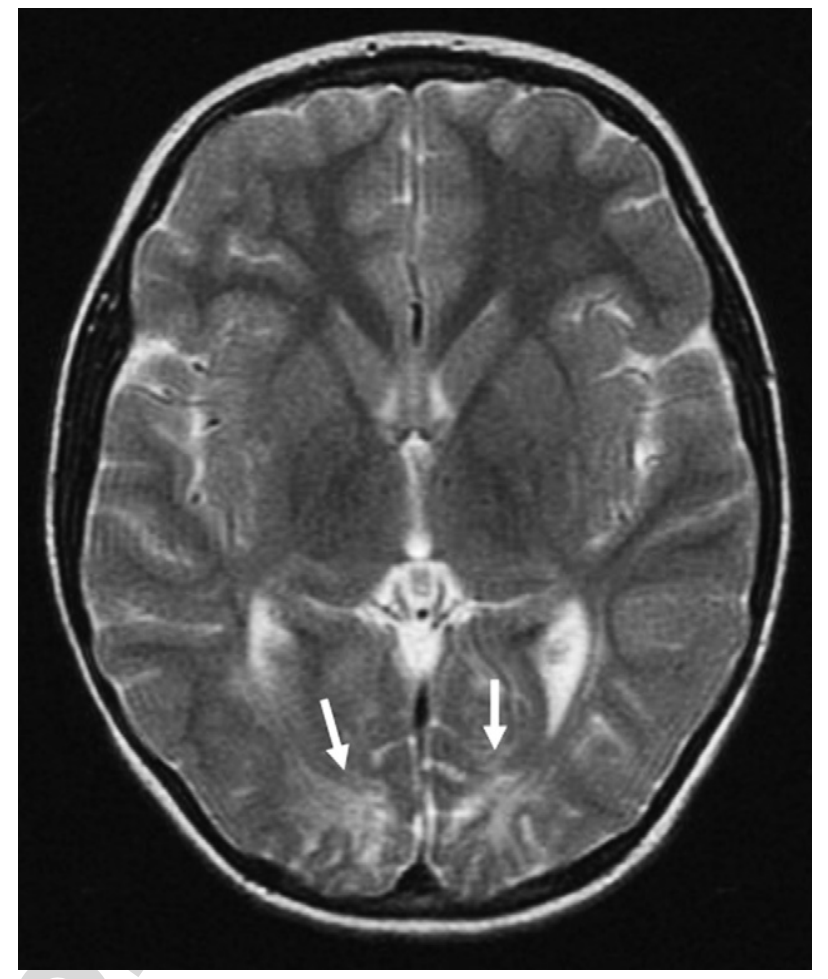

Fig. 2. Axial FSE T2 MRI. Areas of abnormal high signal intensity on T2 weighted MR images are seen in the deep and subcortical periventricular white matter of the occipital and parietal lobes (arrows). white

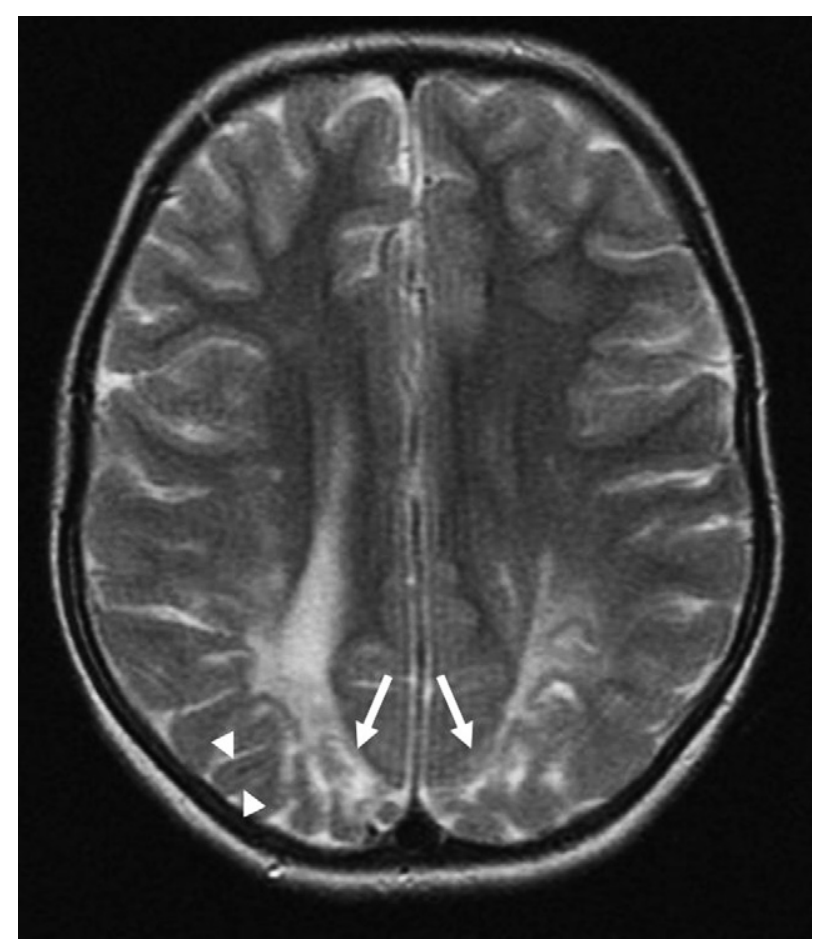

Fig. 3. Axial FSE T2 MRI. Areas of abnormal high signal intensity on T2 weighted MR images are seen in the deep and subcortical periventricular white matter of the occipital and parietal lobes (arrows). There is marked thinning of the occipital and parietal cortex (arrowheads). 


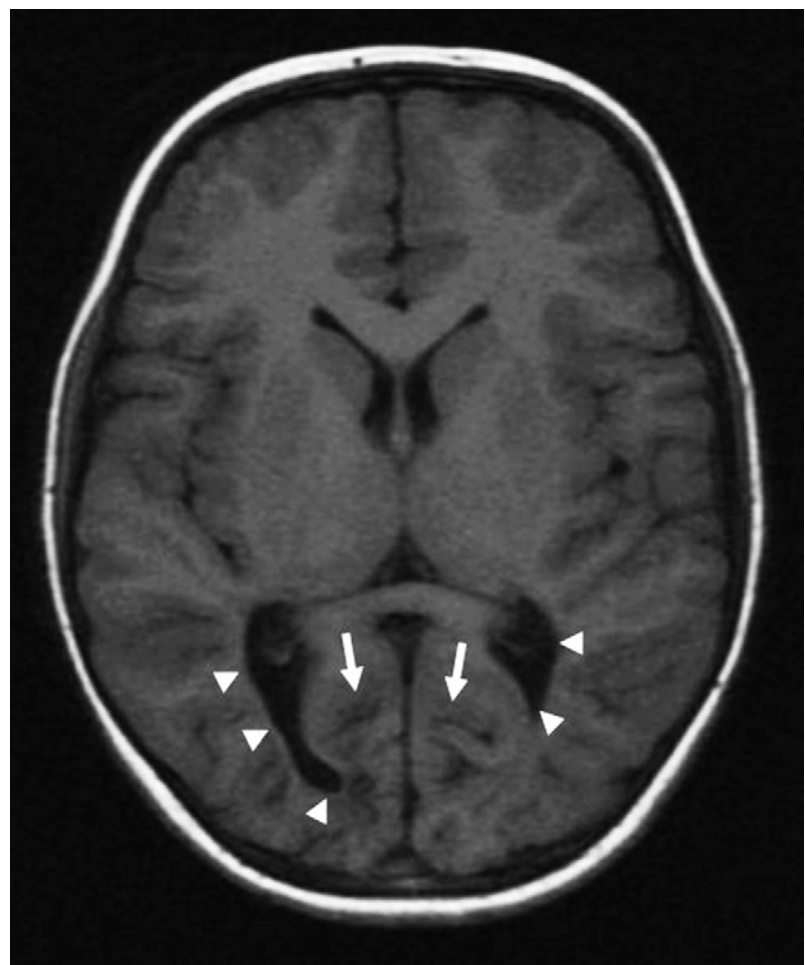

Fig. 4. Axial FSE T1 MRI. The affected areas in the deep and subcortical white matter in the occipital and parietal lobes demonstrate hypointensities on T1 weighted MR images (arrows). The posterior horns of the lateral ventricles are dysmorphic and enlarged (arrowheads), due to tissue loss in the parietal and occipital cortex.

cortex resulting in dysmorphic and enlarged posterior horns of the lateral ventricles, compatible with tissue loss. The basal ganglia and cerebellum appear normal. There is no deviation of the midline.

\section{Discussion}

Persistent hyperinsulinemic hypoglycemia of infancy is a clinically, pathologically and genetically diverse syndrome referring to a group of genetic disorders that result in impaired regulation of insulin secretion in the $\beta$-cells of the pancreas [1]. It is a very rare disease with an estimated annual incidence of $1 / 30,000$ to $1 / 50,000$ live births. In isolated communities with high rates of consanguinity the incidence is much higher and may affect as many as 1 in 2500 live births, especially in the Arabian peninsula [2,5]. Although the majority of the cases occur sporadically, several genetic defects have been described $[1,5,8]$. Two clinically indistinguishable forms of the disease have been identified by pathologists: a diffuse and a focal form.

Hypoglycemia is not uncommon and episodes of hypoglycaemia can be seen in up to $8 \%$ of low-risk neonates $[3,6,9]$. Hypoglycemia during the neonatal period can result from a variety of metabolic and endocrine disorders of fasting adaptation [1,2]. Persistent hyperinsulinemic hypoglycemia of infancy is the most common cause of persistent or recurrent hypoglycaemia [1-4].

Patients with PHHI are usually born at term with normal or low birth weight. Most cases present within the first few days of life with severe prolonged hypoglycaemia [4], resistant to glucagon therapy. Symptoms are often non-specific and include refusal to feed, irritability, hypothermia, sweating, floppiness, lethargy, somnolence, brief myoclonal jerks, respiratory distress, cyanosis, apnea and seizures $[1,5,6]$. In severe cases of life-threatening hypoglycaemic attacks, coma and neonatal death may occur [5].

Neuroglycopenia in association with PHHI increases the risk of brain atrophy and permanent brain damage [8]. Neurological handicap may affect up to $20 \%$ of infants who have suffered from PHHI [2]. Hypoglycemic injury to brain tissue can cause mental retardation, cerebral palsy, seizure disorder, microcephaly, spasticity and ataxia [1,3,6]. Caraballo et al. describe a series of 15 patients with occipital lobe epileptic syndromes following neonatal hypoglycemia [10]. Severity of the hypoglycaemia and delayed initial treatment are thought to be a factor that increases the risk of developmental delay [1].

MRI is the neuro-imaging technique of choice to detect brain damage in PHHI [9]. In numerous cases of persistent hypoglycaemic hypoglycaemia MRI reveals no abnormalities. During or immediately after the acute episode of prolonged hypoglycaemia, diffuse brain edema may be seen [4]. Traill et al. describe two cases of neonatal hypoglycaemia in which MRI shows edema in the parieto-occipital cortex and underlying white matter in the acute phase and profound atrophy of these regions in the chronic phase [11].

The brain lesions on neuroimaging studies in children with prolonged neonatal hypoglycaemia are rather typical, affecting the parietal and occipital lobes of the cerebral hemispheres most severely. Alkaly et al. reviewed 23 cases of neonatal hypoglycaemia and found that in $82 \%$ the occipital lobes were involved [12]. The damage consists of diffuse bilateral symmetrical lesions exhibiting an atrophic character and involving both the cortex and underlying white matter $[3,4,6]$. In most cases the cerebral white matter changes are mild, Mori et al. however present a case in which this damage was extensively and severely [13]. The lesions are detected on MRI images as hyperintensities on T1-weighted images [9]. In severe cases progressive parenchymal loss is described with predominantly occipital involvement, showing hypointense areas resembling infarction and dilatation of the occipital horns of the lateral ventricles [9]. In some patients the brain damage is more extensive, extending into the posterior portions of the frontal lobes and involving the globus pallidus [3]. Occasionally the deep gray matter structures (putamen, caudate nucleus) may be involved as well [4]. Brain damage seems to be more severe with longer duration of the hypoglycaemic episode [12].

Long-term follow up studies after repeated episodes of hypoglycaemia show diffuse brain atrophy and areas of 
cystic degeneration in the affected parietal and occipital lobes. If focal parenchymal abnormalities were present initially, these may develop into laminar cortical necrosis and/or encephalomalacia-like lesions [3,4]. Spar et al. report parenchymal loss or hypodensities in the occipital regions in the late period [7].

The differential diagnosis of this pattern of neonatal brain damage detected by neuroimaging studies is not very extensive. Severe sagittal sinus thrombosis can cause bilateral paramedian brain injury, however the thrombosed sinus can be identified by MRI. Bilateral posterior cerebral artery compression may cause occipital infarction but the pattern is different than that seen in hypoglycaemic brain injury [3]. Neonatal hypoxic-ischemic injury sometimes causes the same pattern of damage as hypoglycaemic damage.

The parietal-occipital lobe pattern of brain injury in PHHI is nearly identical to that of several other causes of hypoglycaemia [3,4]. This suggests that the pattern of damage principally reflects injury from hypoglycaemia and not that of an underlying disorder such as anoxic-ischemic encephalopathy. However delineation of hypoglycaemic brain injury as a distinct condition is complicated as hypoxia and/or asphyxia are frequently coinciding [3,4]. Hypoxia and/or asphyxia potentiate the deleterious effects of hypoglycaemia on the neonatal brain, and vice versa [4]. Moreover in hypoxicischemic injury there is diminished cardiac output, leading to ischemia and resultant lack of oxygen and glucose delivery to the brain. Thus, ischemic and hypoglycaemic injury are superimposed [3].

The cause of the changes in $\mathrm{T} 1$ and $\mathrm{T} 2$ signal intensities on MRI arising from the damaged cerebral cortex is most likely the same as in hypoxic-ischemic injury. Likely possibilities include calcification, petechial hemorrage and myelin degradation [3]. Hypoxic/ischemic conditions pathologically represent reaction of glial cells and macrophages to hypoxia [9]. Neuropathological observations suggest that the most superficial layers of the cortex are primarily involved in hypoglycaemia-induced cortical injury, whereas predominantly the intermediate and deep layers of the cortex are affected in hypoxemic lesions. Lesions in the 'watershed' areas are characteristically in hypoxia-ischemia [11]. Unfortunately currently available spatial resolution of MRI does not allow imaging differentiation of these histopathological changes [4].

Differences in the pattern of hypoglycaemic versus hypoxic-ischemic injury are probably due to the ability of the brain to utilize alternative energy sources (f.e. lactate) and the maintenance of cardiac output and cerebral perfusion in the case of hypoglycaemia. Glucose is the primary energy source for the brain and must be supplied continuously, because the brain has little or no reserve stores of glycogen. This makes the brain vulnerable to damage in cases of hypoglycaemia. Hyperinsulinism is especially likely to cause hypoglycaemic brain damage, because insulin blocks the production of alter- native fuels that might sustain brain metabolism, such as ketones $[1,14]$.

The reason that the parietal and occipital lobes are preferentially affected is not obvious $[3,6,12,14]$. The pattern of damage does not correspond to the regions with the highest glucose metabolism, indicating that it is not a simple matter of demand and supply of glucose as an energy source $[3,6]$. Some investigators postulated that selective occipital vulnerability may be related to intense axonal migration and synaptogenesis, which occurs within the occipital lobes during the neonatal period $[7,9,12,15]$. According to Alkaly et al. during the neonatal period the posterior circulation structures of the brain are more metabolically active than those of the anterior circulation [12]. It has also been hypothesized that high metabolic turnover during early life predisposes this area to injury as occurs in mitochondrial diseases [10]. Other investigators suggest that additional contributing factors may relate to a regional developmental deficit in expression or function of the glucose membrane transporter proteins [14].

\section{References}

[1] Steinkrauss L, Lipman TH, Hendell CD, et al. Effects of hypoglycemia on developmental outcome in children with congenital hyperinsulinism. J Pediatr Nurs 2005;20(2):109-18.

[2] Lindley KJ, Dunne MJ. Contemporary strategies in the diagnosis and management of neonatal hyperinsulinemic hypoglycaemia. J Earl Hum Dev 2005;81:61-72.

[3] Barkovich JA, Ali FA, Rowley HA, Bass N. Imaging patterns of neonatal hypoglycemia. AJNR 1998;19:523-8.

[4] Patay Z. Metabolic disorders. In: Tortori-Donati P, editor. Pediatric neuroradiology brain, vol. 651. Springer; 2005. p. 696-7.

[5] Kaczirek K, Niederle B. Nesidioblastosis: an old term and a new understanding. World J Surg 2004;28:1227-30.

[6] Avenarius HA, Versteege CWM, Engelshove HA. Cerebral damage due to neonatal hypoglycaemia. JBR-BTR 2003;86:136-7.

[7] Spar JA, Lewine JD, Orrison WW. Neonatal hypoglycemia: CT and MR findings. AJNR 1994;15:1477-8.

[8] Nachum Z, Ben-Shlomo I, Rakover Y, et al. Early brain atrophy in persistent hyperinsulinemic hypoglycaemia of infancy. J Pediatr 2002;141(5):706-9.

[9] Kinnala A, Rikalainen H, Lapinleimu H, et al. Cerebral magnetic resonance imaging and ultrasonography findings after neonatal hypoglycemia. Pediatrics 1999;103:724-9.

[10] Caraballo RH, Sakr D, Mozzi M, et al. Symptomatic occipital lobe epilepsy following neonatal hypoglycaemia. Pediatr Neurol 2004;31:24-9.

[11] Traill Z, Squier M, Anslow P. Brain imaging in neonatal hypoglycaemia. Arch Dis Child Fetal Neonatal Ed 1998;79:F145-7.

[12] Alkaly AL, Flores-Sarnat L, Sarnat HB, et al. Brain imaging findings in neonatal hypoglycaemia: case report and review of 23 cases. Clin Pediatr 2005;44:783-90.

[13] Mori F, Nishie M, Houzen H, et al. Hypoglycemic encephalopathy with extensive lesions in the cerebral white matter. Neuropathology 2006;26:147-52.

[14] Filan PM, Inder TE, Cameron FJ, et al. Neonatal hypoglycaemia and occipital injury. J Pediatr 2006;148:552-5.

[15] Aslan Y, Dinc H. MR findings of neonatal hypoglycemia. AJNR 1997;18:994-6. 